\title{
Comparing Quantitative Results of Zeta Factor Method using Various Values of Ionization Cross-sections
}

\author{
Masaki Morita ${ }^{1}$ and Ichiro Onishi ${ }^{1}$ \\ 1. 3-1-2 Musashino, Akishima, Tokyo, Japan, JEOL Ltd.
}

The accuracy of a quantitative analysis in TEM-EDS is generally worse than that in SEM-EDS. There are two main reasons. One is the sample condition and orientation. The sample needs to satisfy uniformities of composition and thickness in a region of electron irradiation. Another reason is an inaccuracy of ionization cross-section used in quantifications such as the Cliff-Lorimer [1] and the Zeta factor [2]. There are some theoretical ionization cross-section tables available for the K line X-ray series. However, the result of quantitative analysis fluctuates depending on the values of ionization crosssection used in the calculation. A $k$ factor of the Cliff-Lorimer method and a zeta factor of the Zeta factor method can be calibrated to obtain better quantitation. However, it is difficult to obtain thin standard specimen for a target sample. Especially, in Cliff-Lorimer method, the sample required to be no significant absorption of generated X-rays. Hence, it's difficult to calibrate these factors and therefore these factors obtained from experiments must be calculated from the theoretical ionization cross-section.

Here we present the results on our attempt to determine the most accurate ionization cross-section by comparing different Zeta factor methods of some samples, experimentally. The Cliff-Lorimer method was not used in this comparison because we cannot determinate the errors of sample thickness and density.

Table 1 shows the comparison of the quantifications by Zeta factor method on different ionization crosssections. The EPMA result is from a bulk sample of San Carlos Olivine, analysed before making the thin sample. The solid angles were calculated by the Zeta factor method from a NiOx standard sample (Ted Pella Inc). Quantitative values are calculated by Zeta factor method on San Carlos Olivine using calculated solid angles. These EDS spectra were taken with a JEM-2100 equipped with $100 \mathrm{~mm}^{2}$ SDD. Accelerating voltage was $200 \mathrm{kV}$ and probe current was $53 \mathrm{pA} . \chi^{2}$ is sum of squared residuals based on EPMA result. The solid angle and quantitative values fluctuate depending on the ionization cross-section values used. The difference of the solid angles shows one of absolute ionization cross-section. In this sample, the quantitative values of Paterson's ionization cross-section were the closest to the EPMA result and this ionization cross-section has good accuracy for the Olivine.

The PAP ionization cross-section was optimised for low accelerating voltage like EPMA. The other ionization cross-sections were calculated by fitting some measured data with Bethe model in TEM. However, the results from these three ionization cross-sections were different and we found that the Paterson's ionization cross-section has the best accuracy, experimentally. 
References:

[1] G. Cliff and G.W. Lorimer, J. Microsc. 103 (1975).

[2] M. Watanabe and D.B. Williams, J. Microsc. 221 (2006).

[3] J.H. Paterson et al, ,J. Microsc. 154 (1989).

[4] N.J. Zaluzec in "Analytical Electron Microscopy", ed. D.B. Williams and D.C. Joy, (San Francisco, San Francisco) p.279-284.

[5] J.L. Pouchou and F. Pichoir in "Electron Probe Quantification”, ed. K.F.J. Heinrich, D.E. Newbury, (Plunum, New York) p.31-75.

[6] C. Jakoby et al, J. de Physiq. Colloq., C9 (1987)

Table 1. Comparison of zeta factor results. Sample for solid angle and quantitative results is NiOx and Olivine, respectively.

\begin{tabular}{|l|c|c|c|c|c|c|}
\hline & Solid angle & \multicolumn{6}{|l|}{ Quant. values [mass\%] } & \\
\cline { 3 - 6 } & [sr] & $\mathbf{O}$ & $\mathbf{M g}$ & $\mathbf{S i}$ & $\mathbf{F e}$ & \multirow{2}{*}{$\chi^{2}$} \\
\hline EPMA & & 57.1 & 25.4 & 14.3 & 3.1 & \\
\hline Paterson J.H. [3] & 0.68 & 55.3 & 25.7 & 14.4 & 4.6 & 5.6 \\
\hline Zaluzec N.J. [4] & 0.49 & 52.9 & 26.2 & 15.2 & 5.7 & 25.9 \\
\hline Jakoby [5] & 0.46 & 52.8 & 26.5 & 15.3 & 5.4 & 26.0 \\
\hline PAP [6] & 0.59 & 51.9 & 26.4 & 15.6 & 6.1 & 38.7 \\
\hline
\end{tabular}

